Message from the Editor

\title{
Avarice, evidence, and the media
}

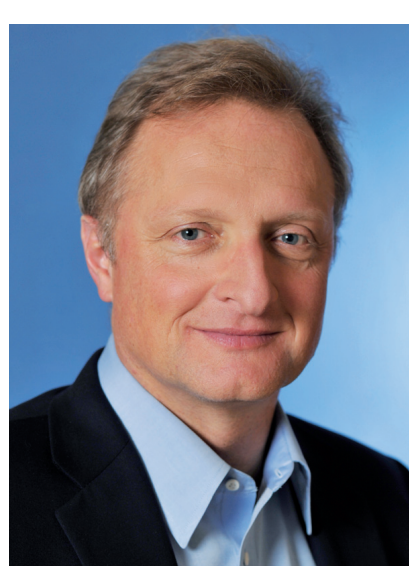

Jens Chapman
Spinal fusion is a hot topic. All one has to do is look at recent headlines (December 2010/ January 2011) from the online versions of The Wall Street Journal, Business Week, Bloomberg BusinessWeek, and others to get a summary of the "media-evidence" on the effectiveness of spinal fusion and the problems associated with it [1-4]:

- Media-evidence conclusion No. 1: There is no evidence that spinal fusion helps patients; they end up taking pain medications for the rest of their lives and cannot work or function.

- Media-evidence conclusion No. 2: While healthcare costs are rising astronomically, billions of dollars are spent on unnecessary surgeries (like fusions).

- Media-evidence conclusion No. 3: Avarice on the part of surgeons and manufacturers is the primary motivation for doing fusion surgery for low back pain.

We are all very familiar with the problems of such reports: inaccurate quotations and/ or misunderstanding of the studies and data they present; emphasis on reports of failed cases and the almost viral spread of the same information from publication to publication that propagates the myths and misinformation.

The motives for spreading these myths are multifold: some media-outlets are primarily interested in circulation, others more in page hits and ad sales. Additionally, there are those with political agendas and others driven by an almost creed-based fervor.

"Myth busting" - in contrast—is a far more arduous task with limited or no chance to gain the public's attention short of embarking in expensive major litigation. Just try to find retractions published in major news media, they are never close to the font size of the original story and are far more likely to be placed near the obituaries or cartoons rather than the front pages where these stories usually originated. This is all the more a factor in electronic media where retractions and corrections are all but irrelevant.

Media reports, such as the ones referenced above, are nothing but escalating salvoes in an increasingly bitter war for the moral high ground for the right to claim back pain as their rightful domain: Medicine-so the shocking headlines suggest—has failed patients, now law (politicians/lawyers/government) and order (insurers with regulations/pathways) must take over to prevent abuse of suffering patients.

Increasingly, politicians, third-party payers, and governmental healthcare agencies are using media reports and the perceived endangerment of public interest to get involved in the healthcare business and are seeking to standardize medical care by creation of guidelines, best practices, clinical pathways, and frank legislation under the guise of "evidence-based medicine." Meaningful answers, however, remain in woefully short supply: As much as politicians would love for it to be the case, one cannot just wish severe recalcitrant low back pain went away, or ignore it, since it defies medications and is certainly immune to legislations.

When discussing the shortcomings of low back pain surgery it helps to remember that from 1999-2006 the number of poisoning deaths nearly doubled in the United States (from about 20,000 to about 37,000), coincident with a near-quadrupling use of prescription opioids [5]. It is safe to say that low back pain played a major role in these statistics. 


\section{References}

1. Waldman P, Armstrong D Highest-paid U.S. doctors get rich with fusion surgery debunked by studies. Available at: http://tinyurl.com/63d7 6ru. Accessed December 29, 2010.

2. Waldman P, Armstrong D Highest-paid U.S. doctors get rich with fusion surgery debunked by studies. Available at: http://tinyurl.com/5rpk fvy. Accessed December 29, 2010.

3. Carreyrou J, McGinty T Top spine surgeons reap royalties, Medicare bounty. Available at: http://tinyurl. com/6xunbca. Accessed: December 20, 2010.

4. Salamon $\mathbf{M}$ Spinal fusion surgery may leave some back pain patients worse off. Available at: http://tinyurl. com/4axjzvz. Accessed February 23, 2011.

5. Coolen P, et al (2009) Overdose deaths involving prescription opioids among Medicaid enrollees. Washington, 2004-2007. MMWR Morb Mortal Wkly Rep; 58:1171-1175.

6. Deyo RA, Mirza SK, Turner JA, et al (2009) Overtreating chronic back pain: time to back off? J Am Board Fam Med; 22:62-68.
The futility of other forms of nonoperative low back pain care has also been pointed out in several landmark studies [6]. Prior to surrendering to the futility of the proposed nihilism, we owe it to our patients to look for more constructive answers.

\section{Let evidence speak for itself}

What ultimately matters is how well our patients do. It is up to us to always seek to do the very best for our patients and document beyond assail what we did and how our patients fared. We, the global community of spine care professionals, need to be aware of the evolution of evidence and actively contribute to enhancing its quality. This is an integral part of EBSJ's mission. I see our immediate charges as follows:

- Respond to patient questions with accurate information and actively correct misinformation among colleagues in other disciplines

- Select the most appropriate patients for a given procedure, based on evidence as it evolves

- Demonstrate the value of procedures with higher-quality evidence

The intricacies of spine care research are not understood by journalists, politicians, or insurance brokers. We cannot expect that or change it. What we can do, is gather evidence in an upfront and open fashion, evaluate and report it without bias, and in response to our findings constantly evolve in how we do things. How adept are we really at critical appraisal of our own evidence, designing studies that keep bias low, and accurately and concisely reporting study findings? Are there aspects of our own research that we've missed?

\section{Lack of clarity in the literature}

The controversy surrounding fusion (and other spine procedures) is in part due to lack of clarity in the literature regarding which patients may benefit most and which may benefit least from such procedures. While some current studies show that, overall, fusion may not be associated with significantly better outcomes compared to nonoperative care options; heterogeneity across patient populations with respect to a variety of factors (eg, type of abnormality, psychosocial concerns, even workers compensation) may profoundly influence the results. Evaluation of such heterogeneity may provide important insights into those who may benefit least and most from fusion for chronic low back pain. This issue of EBSJ features an article on this very topic and is a must read for anyone interested in low back pain fusion analysis.

Well-designed and well-written studies and analyses are needed to counter current controversies and to evolve the current evidence. Methodologically rigorous studies will help identifying subpopulations of patients who may benefit most and those who may not benefit from the treatment. This will help us counsel our patients, select them appropriately, and may provide important evidence for reimbursement decisions eventually.

We can certainly decry, belittle, and then discuss the various media reports, but while we may feel better for venting, there will be little or no impact on the public perceptions or, more importantly, on patient care. Myth busting is not easy. We cannot affect some of the perceptions created by reports like those described above but we can and must occupy the "high ground", continue to stay abreast of evolving evidence, incorporate it into our clinical decision making, and do what we can to enhance the quality of future research. We owe this to the standards set forth in our profession as medical doctors, and most of all we owe it to our patients.

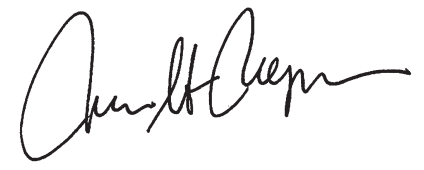

Jens Chapman, Editor-in-Chief 